\title{
Click Chemistry for the Assembly of Gold Nanorods and Silver Nanoparticles
}

\author{
Erica Locatelli, ${ }^{[a]}$ Guido Ori, ${ }^{[b]}$ Marc Fournelle, ${ }^{[\mathrm{c}]}$ Robert Lemor, ${ }^{[\mathrm{c}]}$ \\ Monia Montorsi, ${ }^{[b]}$ and Mauro Comes Franchini*[a]
}

\author{
Dedicated to Professor Giuseppe Bartoli on the occasion of his 70th birthday in recognition of his fruitful career
}

The synthesis of compact nanostructures with highly integrated functionalities through the controlled assembly of nanoparticles (NPs) is potentially of broad interest in research fields such as drug delivery, multimodal imaging, and electronic devices. ${ }^{[1]}$ This concept seems to be particularly important in view of the emerging concept of theranostic, ${ }^{[2]}$ according to which both therapeutic and diagnostic capabilities can be present in two nanostructures. A key step, however, is how to combine individual nanostructures without loosing the original properties. ${ }^{[3]}$

Most often a single type of nanoentities have been assembled by using polydentate or bioconjugate ligands ${ }^{[4]}$ whereas also heterodymer structures ${ }^{[5]}$ composed of quantum dots, magnetic, and metallic NPs have been deeply investigated. In the latter case the optical, magnetic, and electronic properties are often altered or lost, ${ }^{[4 \mathrm{~d}-\mathrm{e}]}$ since the different components are touching each other. An elegant solution is to build multifunctional NPs in which the individual components are spatially separated by an organic framework obtained after an organic reaction. O'Brien ${ }^{[6]}$ recently reported the covalent assembly of $\mathrm{Ag}, \mathrm{Au}$, and CdS spherical NPs through amido and azo linkages. They have demonstrated that controlled growth can be achieved by reacting the NPs at high dilutions showing for the first time, to the best of our knowledge, the formation of covalently bound NP assemblies. Noble metal nanostructures, in particular gold an silver, play a prominent role in nanotechnology. ${ }^{[7]}$ Gold nanorods (GNRs) thanks to the two plasmon absorption bands, tunable from the visible to the near-IR region of the electromagnetic spectrum, have important advantages for applications in biological sensing, imaging, and therapy. ${ }^{[8]}$ The bactericidal and bacteriostatic properties of silver ion have been well known for some time ${ }^{[7 b]}$ but recently Ag NPs have also attracted a great deal of attention in biomedical applications due to their toxicity on cell membranes. ${ }^{[9]}$ Accordingly, it would be important to have a quick, reliable, and simple organic transformation to link these two metallic nanostructures to obtain a theranostic nanostructured system. Moreover, the possibility to build polyethyleneglycol (PEG)-based targetable nanostructures containing GNRs linked to Ag NPs could be particularly useful for nanomedicine applications. This seems to be the case for using a chemistry tailored to generate a new nanoentity quickly and reliably by joining small modular units therefore corresponding to the click chemistry philosophy. ${ }^{[10]}$ One of the most popular reactions within the click chemistry concept is the azide/alkyne Huisgen cycloaddition. ${ }^{[1]}$ We earlier have reported ${ }^{[12,13]}$ of lipophilic GNRs entrapped into PEG-based polymeric NPs and their suitability as optoacoustic contrast agents. ${ }^{[12]}$ Herein, we have investigated how the chemical assembly of GNRs with silver nanoparticles influences the optical properties.

Therefore, we wish to report the first example of click chemistry for the covalent assembly of GNRs with spherical Ag NPs, their entrapment into water-soluble polymeric PEG-based micelles, and the demonstration that this assembled nanostructure is suitable for optoacustic imaging. The new disulfides 1-2 proved to be very efficient for the coating of the two nanostructured surfaces of GNRs and of AgNPs (Figure 1). Indeed, GNRs-CTAB and its ligand exchange [a] E. Locatelli, Dr. M. Comes Franchini

Dipartimento di Chimica Organica "A. Mangini"

University of Bologna

Viale Risorgimento 4, 40136 Bologna (Italy)

Fax: (+39) 0512093654

E-mail: mauro.comesfranchini@unibo.it

[b] Dr. G. Ori, Dr. M. Montorsi

Department of Materials and Environmental Engineering

University of Modena e Reggio Emilia

Via Vignolese 905A, 41100 Modena (Italy)

[c] Dr. M. Fournelle, Dr. R. Lemor

Fraunhofer IBMT, Group Biomedical Ultrasound Research Ensheimer Str. 48, 66386 Sankt Ingbert (Germany)

Supporting information for this article is available on the WWW under http://dx.doi.org/10.1002/chem.201101027.
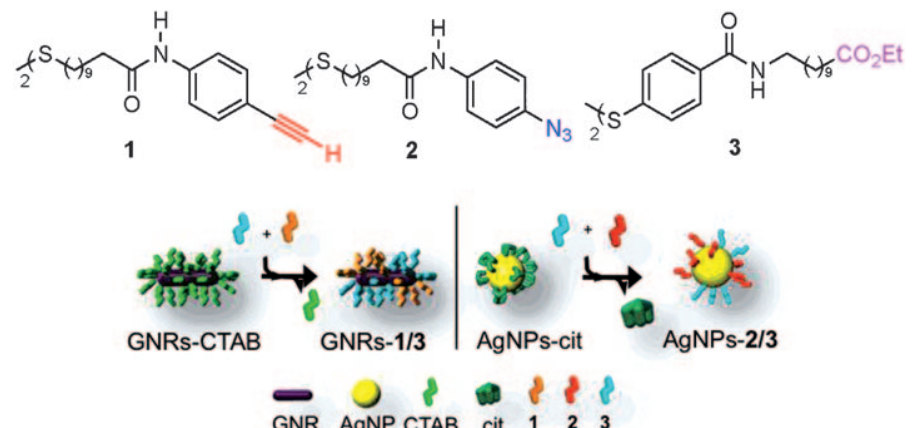

Figure 1. $\omega$-Functionalized disulfides 1-3 used for the coating of the surfaces and lipophilic GNRs-1/3 and AgNPs-2/3. 
with disulfide $\mathbf{1}$ to give lipophilic GNRs-1 was achieved using our reported methods ${ }^{[13]}$ and this procedure was also used for the coating of commercially available Ag NPs with disulfide 2 giving AgNPs-2 (see Figures S1-S4 in the Supporting Information). On the other hand, disulfide $\mathbf{3}$ has been also already reported by us for the coating of GNRs ${ }^{[13]}$ As reported by O'Brien, ${ }^{[6]}$ the concept of high dilution of solvent appears to be necessary for the covalent linkage of the reactive groups that are in the outer shell of NPs but, in our case, we decided to dilute the functional group onto the surface making a coating with a mixture of ligands. This protocol is scarcely reported in the literature. ${ }^{[14]}$

Therefore, GNRs-CTAB were treated at the same time with ligands $\mathbf{1}$ and $\mathbf{3}$ in DMF in different ratios to optimize the coating. UV/Vis spectra $\left(\lambda_{\max }\right.$ at $\left.756 \mathrm{~nm}\right)$ and High Resolution Transmission Electron Microscopy (HRTEM) images of GNRs-1/3 show that the shape of the GNRs has been retained (Figure 2A and B). The presence of the two ligands 1

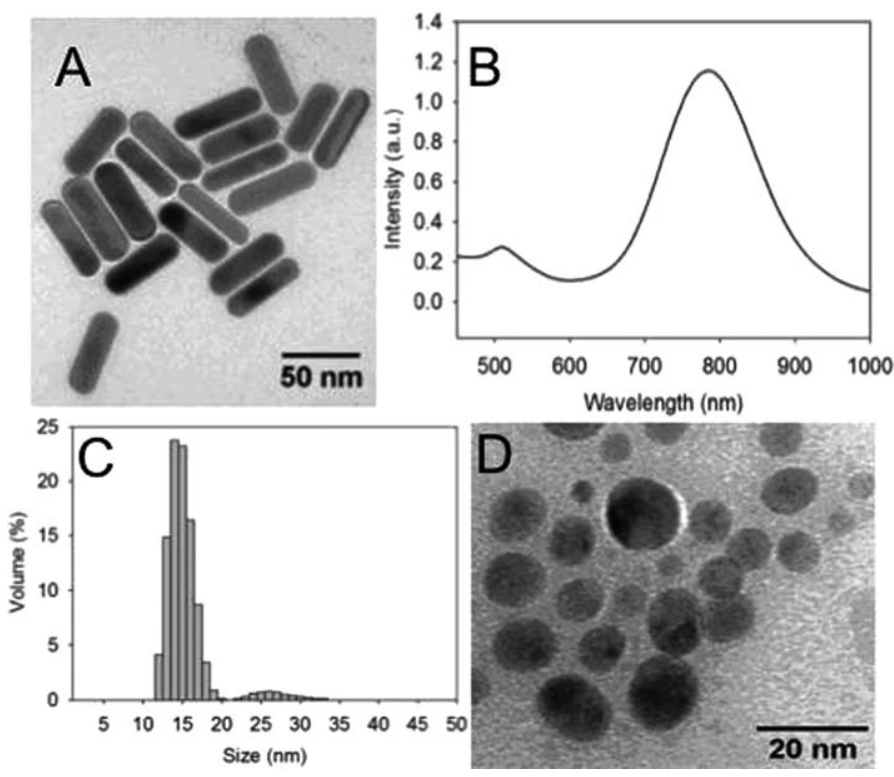

Figure 2. A-B) Transmission electron microscopy (TEM) and UV/Vis of GNRs-1/3. C-D)TEM and DLS image of AgNPs-2/3.

and 3 was further confirmed by ${ }^{1} \mathrm{H}$ NMR spectrum of GNRs-1/3 which shows the signals corresponding to the two ligands, such as the $\mathrm{CH}_{2} \mathrm{O}$ of the ethyl ester of $\mathbf{3}$ and the sp $\mathrm{CH}$ triple bond of $\mathbf{1}$. Moreover, ${ }^{1} \mathrm{H}$ NMR spectrum shows the disappearance of CTAB signals indicating a complete ligand exchange. Using a starting ratio of ligands $\mathbf{1 / 3}$ of $1: 1$, we obtained a final ratio onto the surface of GNRs 1:1 calculated by the two diagnostic signals above mentioned (see Figures S5-S10 and in the Supporting Information and the experimental procedures for more details). Also the AgNPs2/3 became lipophilic after contemporaneous ligand exchange with $\mathbf{2}$ and $\mathbf{3}$, and the same morphology of the starting water soluble Ag NPs was retained as shown in the TEM microphotograph. Dynamic Light Scattering (DLS) measurements indicated that the mixture of ligands produce stable and not aggregated dispersions showing a uniform size distribution with average diameter of $12.0 \mathrm{~nm}$ (Figure $2 \mathrm{C}$ and $\mathrm{D}$ ). In the ${ }^{1} \mathrm{H} \mathrm{NMR}$ spectrum no citrate was detected while this time a ratio of $1: 1$ for $\mathbf{2} / \mathbf{3}$ has been calculated in the ${ }^{1} \mathrm{H}$ NMR spectrum starting with a ratio of ligands 2/3 of $5: 1$. The differences in the two coating could be ascribed to the different solubility of the ligands.

Therefore, following our interest for the 1,3-dipolar cycloaddition, ${ }^{[15]}$ we attempted the click 1,3-dipolar cycloaddition between the acetylenic groups of GNRs-1/3 and the azide groups of AgNPs-2/3 to covalently link the two nanostructures. By simply admixing the lipophilic GNRs-1/3 with AgNPs-2/3 in DMF for $24 \mathrm{~h}$ under reflux we obtained GNRs-1/3-click-AgNPs-2/3, as reported in Scheme 1.

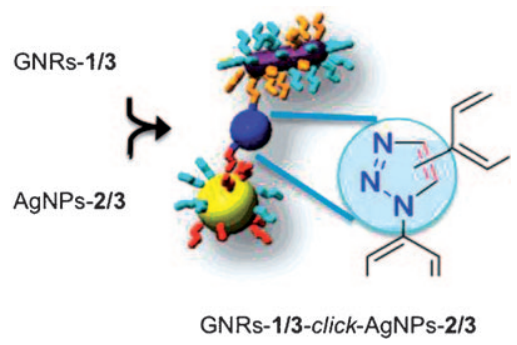

Scheme 1. Synthesis of GNRs-1/3-click-AgNPs-2/3.

The ${ }^{1} \mathrm{H}$ NMR spectrum shows two signals at 8.96 and $9.16 \mathrm{ppm}$ in a 1:1 ratio (Figure 3 ) corresponding to the heteroaromatic $\mathrm{CH}$ of the two possible regioisomers of the 1,2,3triazole ring. Copper and ruthenium are the commonly used

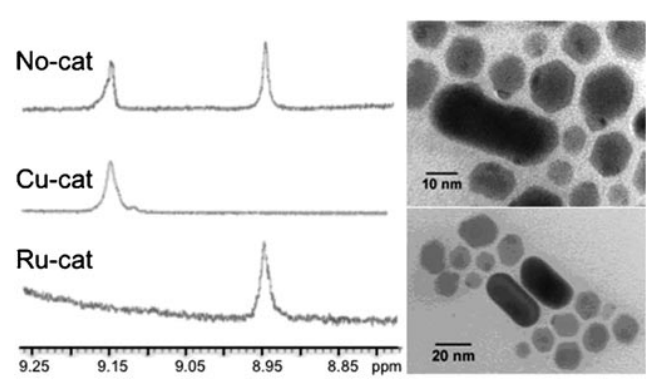

Figure 3. ${ }^{1} \mathrm{H}$ NMR and TEM of GNRs-1/3-click-AgNPs-2/3.

catalysts in the reaction, the use of copper as catalyst results in the formation of 1,4-regioisomer whereas ruthenium results in the formation of the 1,5-regioisomer. Interestingly, under copper catalysis ${ }^{[16]}\left(\mathrm{CuI}\right.$ with $\left.\mathrm{NEt}_{3}\right)$ only the signal at 9.16 was present while the signal at $8.96 \mathrm{ppm}$ was the only detected under ruthenium catalysis with the chloro(pentamethylcyclo pentadienyl)ruthenium(II) tetramer. ${ }^{[17]}$

Figure 3 shows TEM images of the assemblies from a click linkage between GNRs-1/3 and AgNPs-2/3, where GNRs can be easily identify by their peculiar shape. The nature of the two different nanostructures ( $\mathrm{Au}$ and $\mathrm{Ag}$ ) were analyzed and confirmed by energy dispersive analysis 
(EDS; Figures S11 and S12 in the Supporting Information). From TEM images a value of GNR-AgNPs interparticles distance in the range of $2-3 \mathrm{~nm}$ is obtained, calculated between GNRs and the closest AgNPs respect to the GNRs surface, which is in quite good accordance with the value expected (ca. 3 nm, see Figure S13 in the Supporting Information), considering that between the metallic surfaces the organic clicked linkage could be further constrained.

To obtain a further insight on how molecules arrange when assembled around a particle core, we should also invoke the concept of molecular-scale phase separation of a self-assembled monolayer (SAM) formed from a binary mixture of thiols. It has been reported ${ }^{[18]}$ that hydrogenbonding of the buried amides functionality gives formation of nanometer-scale, phase separated discrete domains or islands. This is the typical case that happens when one ligand contains an internal amide functional group and the other is a simple $n$-alkanethiol. In our case, the two ligands $\mathbf{1 / 3}$ for GNRs and 2/3 for AgNPs both contained internal amide functional group but the amide functionalities are in a different position of the alkylic chain, and this would confirm the formation of phase separated discrete domains.

Molecular Dynamics (MD) simulations have been used to obtain a further inside into these aspects: the quantification of the distance between amide groups of ligands 1- and 3SAM respect to an $\mathrm{Au}(111)$ surface and a further analysis of the H-bonding interactions of the systems. The importance of obtaining an insight into mixed-SAM morphology of monolayer-protected nanoparticles have been recently highlighted by the possible effect/role of nanoparticle ligand shell morphology on nanostructuring on a molecular-lengths scale, that can be manipulated in view of specific biomedical application. ${ }^{[19]}$ MD simulations of homogenous-like surface ligands dispositions of $\mathbf{1}$ and $\mathbf{3}$ (60 thiols on $\mathrm{Au}(111)$ in a $\sqrt{ } 3 x \sqrt{ } 3$ arrangement (see Figures $S 15$ and S16 in the Supporting Information) showed the formation of the typical ${ }^{[20]}$ oriented thiol-SAMs respect to the surface $\mathrm{Au}$ normal (Figure 4), thus demonstrating the formation of an evident network of H-bonds between the amide groups for both the ligands.

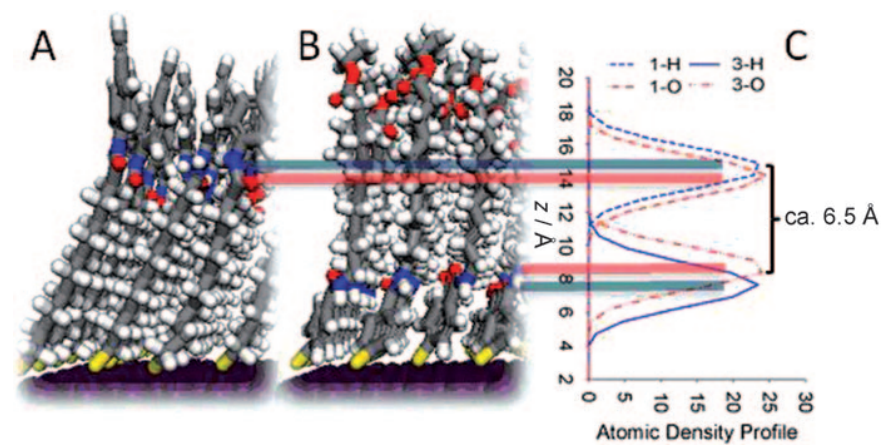

Figure 4. MD snapshots of 1- (A) and 3-SAM (B) on Au 111 surface (Au: violet, S: yellow, C: grey, $\mathrm{H}$ : white, $\mathrm{O}$ : red and $\mathrm{N}$ : blue). C) Atomic density profiles along the $z$-direction of hydrogen and oxygen atoms of 1and 3- amide groups, participating in H-bonding ( $z$ indicates the distance respect to surface Au layer).
Furthermore, the angular distributions analysis showed tilted 1- and 3-chains angles of approximately $29.9^{\circ}$ and $30.5^{\circ}$ (averaged between alkyl and phenyl titled angles, see the Supporting Information), respectively, in good accordance with literature values $\left(\mathrm{ca} .30^{\circ}\right) \cdot{ }^{[20]}$ The atomic density profiles showed averaged interatomic distances of the amide groups respect to Au surface of approximately $14.9 \AA \mathrm{nm}$ and $8.4 \AA$, respectively for $\mathbf{1}$ and $\mathbf{3}$ (Figure 4 ). Thus, the resulting difference (ca. $6.5 \AA$ ) between amide groups heights is significantly high, suggesting the impossibility to promote H-bonding (acceptor-donor distance $\leq 3.5 \AA)^{[21]}$ between 1 and 3 . This would also suggest the formation of phase separated discrete domains in order to promote H-bonding between ligands of the same type.

Polyethyleneglycol (PEG)-based polymeric nanoparticles (PNPs), owing to their stealth character, ${ }^{[22]}$ are useful delivery systems for in vivo applications. Poly(D,L-lactide-co-glycolide)-block-poly(ethylene glycol) copolymer (PLGA- $b$ PEG-COOH) self-assembles to form targetable PNPs (due to the $\mathrm{COOH}$ ) consisting of a hydrophobic PLGA core and a hydrophilic PEG corona-like shell.

The lipophilic cycloadduct was then entrapped into the PLGA- $b$-PEG-COOH using the nanoprecipitation technique $^{[23]}$ as reported in Scheme 2, thus giving click-PNPs. As a comparison we have also prepared polymeric nanoparticles inside, whitout the cycloadducts blank-PNPs (see Figure S14 in the Supporting Information).

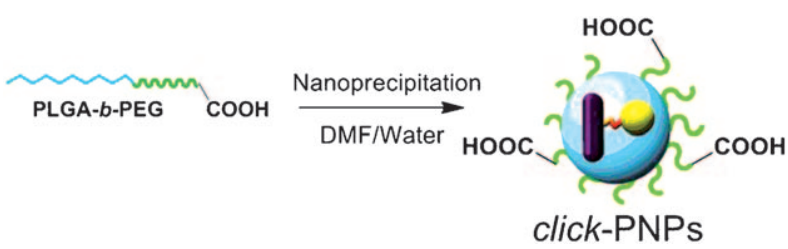

Scheme 2. Nanoprecipitation for the entrapment of GNRs-1/3-clickAgNPs-2/3 into PLGA- $b$-PEG-COOH NPs (click-PNPs).

ICP analysis shows an $\mathrm{Au}$ concentration in the final sample of $15 \mathrm{ppm}$ and simultaneously an Ag concentration of $18 \mathrm{ppm}$. DLS analysis shows that click-PNPs have an uniform size distribution with average diameter of $287.0 \mathrm{~nm}$ and a PDI of 0.10. On the contrary, blank-PNPs have also a PDI of 0.09 but shows an average diameter of $87.3 \mathrm{~nm}$. This increase of size clearly demonstrates the entrapment of GNRs-1/3-click-AgNPs-2/3 into PLGA-b-PEG-COOH nanoparticles. Furthermore, Zeta potential analysis of clickPNPs indicated a value of $-37 \mathrm{mV}$, attesting the stability in water of these nanoparticles.

For proving the suitability of the click-PNPs nanostructure as contrast agent for optoacoustic imaging, we performed a phantom study using particle-loaded alginate structures (see Figure S17 in the Supporting Information).

Conventional GNRs-PNPs, as already reported by $\mathrm{us}^{[13]}$ without Ag-NPs, were used for as reference. Optoacoustic ${ }^{[24]}$ signal amplitudes of phantoms containing either GNRsPNPs or click-PNPs at the same concentration were investi- 
gated using a Nd:YAG laser at its fundamental wavelength of $1064 \mathrm{~nm}$ and an optoacoustic imaging platform (DiPhAS, Fraunhofer IBMT). A reconstructed optoacoustic cross sectional image of the phantoms is shown in Figure 5.

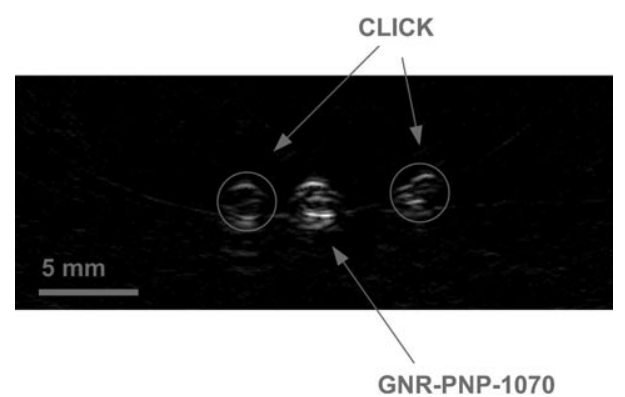

Figure 5. Cross sectional optoacoustic image through 3 alginate spheres loaded with click-PNPs and GNRs-PNPs.

The acquired data shows that click-PNPs particles lead to similar optoacoustic signal amplitudes than conventional GNRs-PNPs without silver NPs. We therefore can state that the chemical linkage between the two nanostructures does not affect the excellent optical and optoacoustical properties of the GNRs entrapped into the polymeric matrix.

The acquired data shows that both click-PNPs and conventional GNR-PNPs are detectable with optoacoustic imaging. In the performed experiments, signals of the clickPNPs were detected with a signal-to-noise ratio of $45 \mathrm{~dB}$ and the GNR-PNPs signals had a SNR of $55 \mathrm{~dB}$ (see Figure 6). Although replacing GNR-PNPs by click-PNPs in the phantoms has resulted in a loss of SNR of $10 \mathrm{~dB}$, we can state that both particles are well suitable as optoacoustic contrast agent due to the high SNR which was observable for both particle types. The chemical linkage between the two nanostructures does therefore only slightly affect the excellent optical and optoacoustical properties of the GNRs entrapped into the polymeric matrix.

In conclusion, we have reported an assembly strategy for metallic nanostructures through the click cycloaddition of

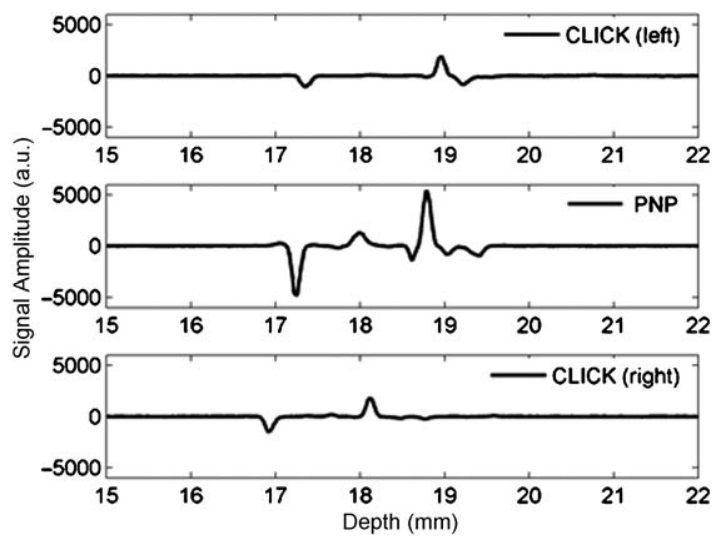

Figure 6. Signal traces of the different nanoparticle loaded phantoms. The signal amplitude is shown as function of depth for the CLICK and the standard GNR-PNPs sphere. lipophilic GNRs with organosoluble Ag NPs. The reaction proceeds cleanly and it is reasonable to expect that the concept of dilution of the functional groups onto nanostructured surfaces, with mixed SAMs, will open new opportunities in nanochemistry. Moreover, our GNRs-1/3-clickAgNPs-2/3 has been entrapped into biodegradable, FDA-approved and targetable PEG-based polymeric nanoaparticles (click-PNPs) and phantom experiments have shown quantitatively that they efficiently generate ultrasound signals after irradiation with laser pulses. Therefore we have shown that the organic linkage with silver nanoparticles did not affect the suitability of the GNRs as contrast agents for optoacoustic imaging. Since the toxicity of AgNPs against cancer cells is a new emerging concept, ${ }^{[9]}$ the click-PNPs herein reported are currently being investigated in our laboratories for theranostic applications in nanomedicine for cancer treatment.

\section{Acknowledgements}

We are grateful to Dr. M. Zapparoli of the CIGS for TEM analysis (University of Modena and R.E., Italy).

Keywords: click chemistry $\cdot$ functionalized disulfides $\cdot$ gold nanorods $\cdot$ nanoparticles $\cdot$ optoacoustic imaging

[1] S.-H. Hu, X. Gao, J. Am. Chem. Soc. 2010, 132, 7234-7237.

[2] a) D. Pan, S. D. Caruthers, G. Hu, A. Senpan, M. J. Scott, P. J. Gaffney, S. A. Wickline, G. M. Lanza, J. Am. Chem. Soc. 2008, 130, 9186-9187; b) C.-H. Jaemoon Yang, H.-J. Ko, J.-S. Suh, H.-G. Yoon, L. Kwangyeol, Y.-M. Huh, H. Seungjoo, Angew. Chem. 2007, 119, 8992-8995; Angew. Chem. Int. Ed. 2007, 46, 8836-8839; c) M. Comes Franchini, G. Baldi, D. Bonacchi, D. Gentili, G. Giudetti, A. Lascialfari, M. Corti, E. Micotti, U. Guerrini, L. Sironi, P. Gelosa, P. Marmorato, J. Ponti, C. Ravagli, A. Ricci, Small 2010, 6, 366-370.

[3] Y. Jin, X. Gao, Nat. Nanotechnol. 2009, 4, 571-576.

[4] a) X. Peng, T. E. Wilson, P. Alivisatos, P. G. Schultz, Angew. Chem. 1997, 109, 113-115; Angew. Chem. Int. Ed. Engl. 1997, 36, 145-147; b) L. C. Brousseau III, J. P. Novak, S. M. Marinakos, D. L. Feldheim, Adv. Mater. 1999, 11, 447-449; c) J. P. Novak, D. L. Feldheim, J. Am. Chem. Soc. 2000, 122, 3979-3980; d) P. Pramod, K. G. Thomas, Adv. Mater. 2008, 20, 4300-4305; e) M. Green, D. Smyth-Boyle, J. Mater. Chem. 2007, 17, 3588-3590.

[5] a) X. Xiang, X. Wu, D. Liu, X. Jiang, W. Chu, Z. Li, Y. Ma, W. Zhou, S. Xie, Nano Lett. 2006, 6, 2290-2294; b) S. Kim, S. K. Kim, S. Park, J. Am. Chem. Soc. 2009, 131, 8380-8381; c) O. M. Wilson, R. W. J. Scott, J. C. Garcia-Martinez, R. M. Crooks, J. Am. Chem. Soc. 2005, 127, 1015-1024; d) C. Xu, B. Wang, S. Sun, J. Am. Chem. Soc. 2009, 131, 4216-4217; e) T. Mokari, E. Rothenberg, I. Popov, R. Costi, U. Banin, Science 2004, 304, 1787-1790.

[6] W. Maneeprakorn, M. A. Malik, P. O'Brien, J. Am. Chem. Soc. 2010, 132, 1780-1781.

[7] a) Gold Chemistry: Applications and Future Directions in the Life Sciences (Ed.: F. Mohr), Wiley-VCH, VCH, 2009; b) S. N. Lakshmi, C. T. Laurencin, J. Biomed. Nanotechnol. 2007, 3, 301-316.

[8] a) C. J. Murphy, A. M. Gole, J. W. Stone, P. N. Sisco, A. M. Alkilany, E. C. Goldsmith, S. C. Baxter, Acc. Chem. Res. 2008, 41, 1721-1730; b) P. K. Jain, X. Huang, I. H. El-Sayed, M. A. El-Sayed, Acc. Chem. Res. 2008, 41, 1578-1586; c) X. Huang, I. H. El-Sayed, W. Qian, M. A. El-Sayed, J. Am. Chem. Soc. 2006, 128, 2115-2120; d) A. M. Alkilany, P. K. Nagaria, C. R. Hexel, T. J. Shaw, C. J. Murphy, M. D. 
Wyatt, Small 2009, 5, 701-708; e) B. P. Khanal, E. R. Zubarev, Angew. Chem. 2007, 119, 2245-2248; Angew. Chem. Int. Ed. 2007, $46,2195-2198$.

[9] a) J. V. Rogers, C. V. Parkinson, Y. W. Choi, J. L. Speshock, S. M. Hussain, Nanoscale Res. Lett. 2008, 3, 129-133; b) Q. Wu, H. Cao, Q. Luan, J. Zhang, Z. Wang, J. H. Warner, A. A. R. Watt, Inorg. Chem. 2008, 47, 5882-5888; c) P. V. Asharani, Y. L. Wu, Z. Gong, S. Valiyaveettil, Nanotechnology 2008, 19, 255102; d) L. S. Nair, C. T. Laurencin, J. Biomed. Nanotechnol. 2007, 3, 301-316; e) E. T. Hwang, H. J. Lee, Y. J. Chae, Y. S. Kim, B. C. Kim, B. I. Sang, Small 2008, 4, 746-750

[10] H. C. Kolb, M. G. Finn, K. B. Sharpless, Angew. Chem. 2001, 113, 2056-2057; Angew. Chem. Int. Ed. 2001, 40, 2004-2021.

[11] R. Huisgen in 1,3-Dipolar Cycloaddition Chemistry (Ed.: A. Padwa), Wiley, New York, 1984.

[12] D. Gentili, G. Ori, M. Comes Franchini, Chem. Commun. 2009, 5874-5876.

[13] M. Comes Franchini, J. Ponti, R. Lemor, M. Fournelle, F. Broggi, E. Locatelli, J. Mater. Chem. 2010, 20, 10908-10914. G. Baldi, D. Bonacchi, M. Comes Franchini, D. Gentili, G. Lorenzi, A. Ricci, C. Ravagli, Langmuir 2007, 23, 4026-4028.

[14] A. C. Templeton, M. J. Hostetler, C. T. Kraft, R. W. Murray, J. Am. Chem. Soc. 1998, 120, 1906-1910.

[15] a) B. F. Bonini, M. Comes Franchini, D. Gentili, E. Locatelli, A. Ricci, Synlett 2009, 2328-2332; b) B. F. Bonini, F. Boschi, M. Comes Franchini, M. Fochi, F. Fini, A. Mazzanti, A. Ricci, Synlett 2006 $543-547$; c) L. Bernardi, B. F. Bonini, M. Comes Franchini, M. Fochi, M. Folegatti, S. Grilli, A. Mazzanti, A. Ricci, Tetrahedron: Asymmetry 2004, 15, 245-250.

[16] V. D. Bock, H. Hiemstra, J. H. van Maarseveen, Eur. J. Org. Chem. 2006, $51-68$

[17] L. Zhang, X. Chen, P. Xue, H. H. Y. Sun, I. D. Williams, K. B. Sharpless, V. V. Fokin, G. Jia, J. Am. Chem. Soc. 2005, 127, 15998-15999.

[18] a) S. J. Stranick, S. V. Atre, A. N. Parikh, M. C. Wood, D. L. Allara, N. Winograd, P. S. Weiss, Nanotechnology 1996, 7, 438-442; b) J. P.
Folkers, P. E. Laibinis, G. M. Whitesides, J. Deutch, J. Phys. Chem 1994, 98, 563-569; c) R. K. Smith, S. M. Reed, P. A. Lewis, J. D. Monnell, R. S. Clegg, K. F. Kelly, L. A. Bumm, J. E. Hutchinson, P. S. Weiss, J. Phys. Chem. B 2001, 105, 1119-1122.

[19] a) A. Centrone, E- Penzo, M. Sharma, J. W. Myerson, A. M. Jackson, N. Marzari, F. Stellacci, Proc. Natl. Acad. Sci. USA 2008, 105 9886-9881; b) A. Hung, S. Mwenifumbo, M. Mager, J. J. Kuna, F. Stellacci, I. Yarovsky, M. M. Stevens, J. Am. Chem. Soc. 2011, 133 1438-1450.

[20] a) R. G. Nuzzo, L. H. Dubois, D. L. Allara, J. Am. Chem. Soc. 1990, 112, 558-569; b) T. E. Dirama, J. A. Johnson, Langmuir 2007, 23 , 12208-12216; c) J. Jia, Y. D. Huang, J. Long, J. M. He, H. X. Zhang, Appl. Surf. Sci. 2009, 255, 6451-6459.

[21] a) G. R. Desiraju, T. Steiner, The Weak Hydrogen Bond in Structural Chemistry and Biology, Oxford University Press, New York, 1999; b) A. Luzar, D. Chandler, Phys. Rev. Lett. 1996, 76, 928-931.

[22] a) R. Gref, Y. Minamitake, M. T. Peracchia, V. Trubetskoy, V. Torchilin, R. Langer, Science 1994, 263, 1600-1603; b) D. E. Owens III, N. A. Peppas, Int. J. Pharm. 2006, 307, 93-102.

[23] a) M. Chorny, I. Fishbein, H. D. Danenberg, G. Golomb, J. Controlled Release 2002, 83, 389-400; b) J. Chenga, B. A. Teplya, I Sherifia, J. Sunga, G. Luthera, F. X. Gua, E. Levy-Nissenbauma, A. F. Radovic-Moreno, R. Langer, O. C. Farokhzad, Biomaterial 2007, 28, 869-876; c) C. Fonseca, S. Simoes, R. E. Gaspar, J. Controlled Release 2002, 83, 273-286; d) B. F. Bonini, C. M. Camaggi, M. Comes Franchini, D. Gentili, A. Pession, M. Rani, E. Strocchi, Eur. J. Med. Chem. 2010, 45, 2024-2033.

[24] A. A. Oraevsky and A. A. Karabutov, Optoacoustic Tomography, Biomedical Photonics Handbook, CRC Press, Boca Raton, FL, 2003, Chapter 34.

Received: April 4, 2011

Revised: May 24, 2011

Published online: July 8, 2011 